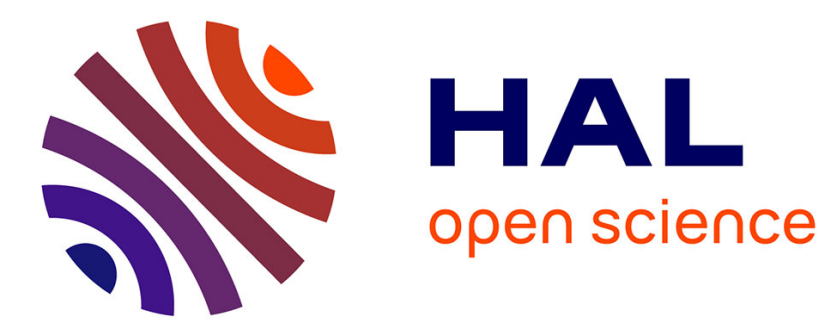

\title{
High gain observer for a class of non-triangular systems
}

Mondher Farza, Mohammed M'Saad, Moncef Triki, Tarek Maatoug

\section{To cite this version:}

Mondher Farza, Mohammed M'Saad, Moncef Triki, Tarek Maatoug. High gain observer for a class of non-triangular systems. Systems and Control Letters, 2010, 60, pp.27-35. 10.1016/j.sysconle.2010.09.009 . hal-01059914

\section{HAL Id: hal-01059914 https://hal.science/hal-01059914}

Submitted on 8 Sep 2014

HAL is a multi-disciplinary open access archive for the deposit and dissemination of scientific research documents, whether they are published or not. The documents may come from teaching and research institutions in France or abroad, or from public or private research centers.
L'archive ouverte pluridisciplinaire HAL, est destinée au dépôt et à la diffusion de documents scientifiques de niveau recherche, publiés ou non, émanant des établissements d'enseignement et de recherche français ou étrangers, des laboratoires publics ou privés. 


\title{
High gain observer for a class of non-triangular systems
}

\author{
M. Farza ${ }^{\mathrm{a}, *}$, M. M'Saad $^{\mathrm{a}}$, M. Triki ${ }^{\mathrm{a}, \mathrm{b}}$, T. Maatoug $^{\mathrm{b}}$ \\ ${ }^{a}$ GREYC, UMR 6072 CNRS, Université de Caen, ENSICAEN, 6 Bd Maréchal Juin, 14050 Caen Cedex, France \\ b ENIS, Département de Génie électrique, BP W, 3038 Sfax, Tunisia
}

Keywords:

Nonlinear systems

MIMO systems

Observability for any input

High gain observers

\section{A B S T R A C T}

This paper presents a high gain observer for a class of MIMO nonlinear systems involving some uncertainties. The latter is particularly composed of cascade subsystems where each subsystem is associated with a subset of the output variables, and assumes a triangular dependence on its own state variables and may depend on the state variables of all other subsystems. The main contribution consists in extending the available results to allow more interconnections between the subsystems. Of fundamental interest, it is shown that the underlying observation error exponentially converges to zero in the absence of uncertainties. Moreover, the observation error can be made as small as desired by properly specifying the observer design parameter in the case where uncertainties are considered.

\section{Introduction}

This paper presents a state observer for nonlinear systems that are diffeomorphic to

$\left\{\begin{array}{l}\dot{x}=A x+\varphi(u, x)+\bar{\varepsilon}(t) \\ y=C x\end{array}\right.$

where the terms are defined as follows.

- $x$ denotes the state of the system and is composed as follows

$$
x=\left(\begin{array}{c}
x^{1} \\
x^{2} \\
\vdots \\
x^{q}
\end{array}\right) \in \mathbb{R}^{n} \quad \text { with } x^{k}=\left(\begin{array}{c}
x_{1}^{k} \\
x_{2}^{k} \\
\vdots \\
x_{\lambda_{k}}^{k}
\end{array}\right) \in \mathbb{R}^{n_{k}}
$$

where $x_{i}^{k}=\left(\begin{array}{c}x_{i, 1}^{k} \\ \vdots \\ x_{i, p_{k}}^{k}\end{array}\right) \in \mathbb{R}^{p_{k}}$ with $x_{i, j}^{k} \in \mathbb{R}$ for $k=1, \ldots, q, i=$ $1, \ldots, \lambda_{k}, j=1, \ldots, p_{k}$ with $\sum_{k=1}^{q} n_{k}=\sum_{k=1}^{q} p_{k} \lambda_{k}=n ; p_{k} \geq$ 1 and $\lambda_{k} \geq 2$

- $u$ and $y$, respectively, denote the input and the output of the system. The output is particularly composed as follows

\footnotetext{
* Corresponding author.

E-mail address: mfarza@greyc.ensicaen.fr (M. Farza).
}

$$
y=\left(\begin{array}{c}
y_{1} \\
y_{2} \\
\vdots \\
y_{q}
\end{array}\right) \in \mathbb{R}^{p}
$$

where $y_{k} \in \mathbb{R}^{p_{k}}$ for $k=1, \ldots, q$ and hence

$$
\sum_{k=1}^{q} p_{k}=p
$$

- The matrices $\mathrm{A}$ and $\mathrm{C}$ are respectively given by

$$
\begin{gathered}
A=\left[\begin{array}{lll}
A_{1} & & \\
& \ddots & \\
& & A_{q}
\end{array}\right] \\
\text { with } A_{k}=\left[\begin{array}{cccc}
0 & I_{p_{k}} & & 0 \\
\vdots & & \ddots & \\
0 & \cdots & 0 & I_{p_{k}} \\
0 & \cdots & 0 & 0
\end{array}\right]
\end{gathered}
$$

and

$$
\begin{gathered}
C=\left[\begin{array}{ccc}
C_{1} & & \\
& \ddots & \\
& & C_{q}
\end{array}\right] \\
\quad \text { with } C_{k}=\left[\begin{array}{llll}
I_{p_{k}} & 0 & \cdots & 0
\end{array}\right] .
\end{gathered}
$$


- $\varphi(u, x)$ denotes the nonlinear function field which is composed as follows

$$
\begin{gathered}
\varphi(u, x)=\left(\begin{array}{c}
\varphi^{1}(u, x) \\
\varphi^{2}(u, x) \\
\vdots \\
\varphi^{q}(u, x)
\end{array}\right) \in \mathbb{R}^{n} \\
\text { with } \varphi^{k}(u, x)=\left(\begin{array}{c}
\varphi_{1}^{k}(u, x) \\
\varphi_{2}^{k}(u, x) \\
\vdots \\
\varphi_{\lambda_{k}}^{k}(u, x)
\end{array}\right) \in \mathbb{R}^{n_{k}}
\end{gathered}
$$

where the function $\varphi_{i}^{k}(u, x) \in \mathbb{R}^{p_{k}}$ is differentiable with respect to $x$ and assumes the following structural dependence on the state variables.

$$
\begin{aligned}
& \text { - For } 1 \leq i \leq \lambda_{k}-1 \text { : } \\
& \varphi_{i}^{k}(u, x)=\varphi_{i}^{k}\left(u, x^{1}, x^{2}, \ldots, x^{k-1},\right. \\
& \left.\quad x_{1}^{k}, x_{2}^{k}, \ldots, x_{i}^{k}, x_{1}^{k+1}, x_{1}^{k+2}, \ldots, x_{1}^{q}\right) . \\
& \text { - For } i=\lambda_{k} \text { : } \\
& \varphi_{\lambda_{k}}^{k}(u, x)=\varphi_{\lambda_{k}}^{k}\left(u, x^{1}, x^{2}, \ldots, x^{q}\right) .
\end{aligned}
$$

- $\bar{\varepsilon}(t)$ is a completely unknown function with the following structure:

$$
\bar{\varepsilon}(t)=\left(\begin{array}{c}
\bar{\varepsilon}^{1}(t) \\
\bar{\varepsilon}^{2}(t) \\
\vdots \\
\bar{\varepsilon}^{q}(t)
\end{array}\right) \in \mathbb{R}^{n} \quad \text { with } \bar{\varepsilon}^{k}(t)=\left(\begin{array}{c}
\bar{\varepsilon}_{1}^{k}(t) \\
\bar{\varepsilon}_{2}^{k}(t) \\
\vdots \\
\bar{\varepsilon}_{\lambda_{k}}^{k}(t)
\end{array}\right) \in \mathbb{R}^{n_{k}}
$$

where the functions $\bar{\varepsilon}_{i}^{k}(t) \in \mathbb{R}^{p_{k}}$ are given by

$$
\bar{\varepsilon}_{i}^{k}= \begin{cases}0 & \text { for } k=1, \ldots, q \text { and } i=1, \ldots, \lambda_{k}-1 \\ \varepsilon_{k}(t) & \text { for } k=1, \ldots, q \text { and } i=\lambda_{k}\end{cases}
$$

where the $\varepsilon_{k}(t)^{\prime}$ 's, $k=1, \ldots, q$ are unknown bounded functions, i.e.

$$
\exists \beta>0 ; \forall t \geq 0 ; \quad\left\|\varepsilon_{k}(t)\right\| \leq \beta .
$$

The observer design problem for nonlinear dynamical systems has received a remarkable attention over the last years. More specifically, four approaches have been pursued to design observers for nonlinear systems. The first approach, which has met a great success in the past, is based on the Kalman filter which is used as a nonlinear observer [1]. The success of such an approach is mainly due to its underlying implementation simplicity independently of the system complexity. Nevertheless, there is a major drawback of this approach that would be worth mentioning, namely, the lack of guaranteed stability. The second approach is that based on linearizable error dynamics where state transformations are exhibited in order to put the considered systems in a form where the nonlinearities depend only on the inputs and the outputs [2-8]. The resulting class of systems constitutes a subclass of system (1) with the particularity that the function $\varphi$ only depends on the input $u$ and output $y$. The third approach is based on the LMI techniques and has been the subject of many studies over the past decade $[9,10]$. The involved systems are composed of a constant linear part and a nonlinear part that is assumed to be globally Lipschitz. The observer gain is determined through the resolution of a LMI problem and thereby the observer design is closely related to the feasibility of the involved LMI problem. The latter is by no means known $a$ priori and is to be determined numerically as pointed out in [11]. The fourth approach is based on the observable canonical forms and uses the concept of observability for any input [12]. This concept rises from the well-known fact that unlike linear systems, the observability of nonlinear systems does depend on the inputs. A necessary and sufficient condition for the observability for any input of single-output-input affine nonlinear systems has been established in [13]. The involved systems are characterized by the well-known triangular form and are included in the class of systems (1) with $p=1$. The triangular canonical form is composed of a fixed linear dynamics component together with a nonlinear triangular controlled one. This canonical form makes it possible to design a high gain observer under some global Lipschitz assumption on the controlled part. The gain of the observer is determined from a usual algebraic Lyapunov equation. Many generalizations of this result to systems with many outputs have been proposed in [14-21]. Other approaches that do not fall in the above four classes can be found in [22-24].

The aim of this contribution is to extend the available results by proposing a canonical form that characterizes a large class of nonlinear systems that are observable for any input. It is worth pointing out two remarks.

- In the absence of uncertainties, the class of systems (1) extends that considered in [19] in two directions. First, each subsystem is allowed to be multi-output unlike in [19] where all the subsystems are single-output. Second, the nonlinearity structure is no longer triangular as in [19]. More specifically, the nonlinearity intervening in the last equation of each subsystem depends on all the state variables. Furthermore, it is worth noticing that the class of systems considered in [21] is diffeomorphic to system (1) with $q=1$. This means that all the outputs belong to the same subsystem in [21].

- In the presence of uncertainties, system (1) includes the class of systems considered in [20]. Recall that many systems involving unknown inputs can be put in the form (1) allowing thereby to design nonlinear observers that simultaneously estimate the state variables and the unknown inputs (see [25] for instance).

This paper is organized as follows. In Section 2, one first shows that system (1) is indeed observable for any input. Then some notations and definitions with a technical lemma are given. Finally, the structure exhibited by system (1) is discussed in order to emphasize the main contributions with respect to the available results. The observer design is developed in Section 3 with a full stability and convergence analysis. Simulation results are given in Section 4 to show the performances of the proposed observer. Finally, concluding remarks are given in Section 5.

\section{Preliminaries}

After showing that system (1) is observable for any input, one introduces some variables used in the observer equations. A particular emphasis is put on a technical lemma needed in the proof of the main result, namely, the convergence of the observation error. Finally, one discusses the structure of the considered class of systems and puts forward some novelties exhibited by this structure in comparison with previous works related to high gain observer design. The difficulties in the observer design raised by the considered structure are emphasized and the technique behind achieving the observer synthesis is roughly explained.

\subsection{Observability for any input}

The time derivative of the $\left(\lambda_{k}-1\right)$ first components of the subsystem $k$, for $k=1, \ldots, q$, can be described as follows:

$\left\{\begin{array}{c}\dot{x}_{i}^{k}=x_{i+1}^{k}+\varphi_{i}^{k}\left(u, x^{1}, \ldots, x^{k-1}, x_{1}^{k}, x_{2}^{k}, \ldots, x_{i}^{k},\right. \\ \left.x_{1}^{k+1}, x_{1}^{k+2}, \ldots, x_{1}^{q}\right), \quad i=1, \ldots, \lambda_{k}-1 \\ y_{k}=x_{1}^{k} .\end{array}\right.$ 
Let $x=\left(\begin{array}{c}x^{1} \\ x^{2} \\ \vdots \\ x^{q}\end{array}\right)$ with $x^{k}=\left(\begin{array}{c}x_{1}^{k} \\ x_{2}^{k} \\ \vdots \\ x_{\lambda_{k}}^{k}\end{array}\right)$ and $\bar{x}=\left(\begin{array}{c}\bar{x}^{1} \\ \bar{x}^{2} \\ \vdots \\ \bar{x}^{q}\end{array}\right)$ with $\bar{x}^{k}=\left(\begin{array}{c}\bar{x}_{1}^{k} \\ \bar{x}_{2}^{k} \\ \vdots \\ \bar{x}_{\lambda_{k}}^{k}\end{array}\right)$ be two trajectories of system (1) associated with the outputs $y=$ $\left(\begin{array}{c}y_{1}=x_{1}^{1} \\ y_{2}=x_{1}^{2} \\ \vdots \\ y_{q}=x_{1}^{q}\end{array}\right)$ and $\bar{y}=\left(\begin{array}{c}\bar{y}_{1}=\bar{x}_{1}^{1} \\ \bar{y}_{2}=\bar{x}_{1}^{2} \\ \vdots \\ \bar{y}_{q}=\bar{x}_{1}^{q}\end{array}\right)$, respectively. In order to show that system (1) is observable (for any input), it suffices to show that:

$y=\bar{y} \Longrightarrow x=\bar{x}$.

Indeed, suppose that $y=\bar{y}$. Then, one obviously has

$x_{1}^{k}=y_{k}=\bar{y}_{k}=\bar{x}_{1}^{k} \quad$ for $k=1, \ldots, q$.

In particular, for $k=1$, one has

$x_{1}^{1}=\bar{x}_{1}^{1}$.

Differentiating each term of Eq. (8) with respect to time gives

$$
\begin{aligned}
x_{2}^{1}+\varphi_{1}^{1}\left(u, x_{1}^{1}, y_{2}, \ldots, y_{q}\right) & =\bar{x}_{2}^{1}+\varphi_{1}^{1}\left(u, \bar{x}_{1}^{1}, \bar{y}_{2}, \ldots, \bar{y}_{q}\right) \\
& =\bar{x}_{2}^{1}+\varphi_{1}^{1}\left(u, x_{1}^{1}, y_{2}, \ldots, y_{q}\right) .
\end{aligned}
$$

The last equality comes from (7) and yields

$x_{2}^{1}=\bar{x}_{2}^{1}$.

Differentiating again each term of Eq. (10) with respect to time and taking into account the triangular structure of $\varphi_{2}^{1}$ with respect to $y$ and $x_{2}^{1}$ yields

$x_{3}^{1}=\bar{x}_{3}^{1}$.

Repeating the same process until $i=\lambda_{1}-1$ yields $x_{\lambda_{1}}^{1}=\bar{x}_{\lambda_{1}}^{1}$. Thus, one has $x^{1}=\bar{x}^{1}$. Note that the last equation of the block (corresponding to $i=\lambda_{1}$ ) is not used. Now, it suffices to use the process described above for the remaining subsystems, for $k=$ $2, \ldots, q$, from the first equation of the subsystem associated with $x_{1}^{k}$ until the equation associated with $x_{\lambda_{k}-1}^{k}$. At the end of each stage, one gets $x^{k}=\bar{x}^{k}$; the equality between both trajectories $x$ and $\bar{x}$ is established at the last stage corresponding to the block $q$.

\subsection{Some definitions and notations}

For $k=1, \ldots, q$, let $\Delta_{k}(\theta)$ be the diagonal matrix defined by:

$\Delta_{k}(\theta)=\operatorname{diag}\left(I_{p_{k}}, \frac{1}{\theta^{\delta_{k}}} I_{p_{k}}, \ldots, \frac{1}{\theta^{\delta_{k}\left(\lambda_{k}-1\right)}} I_{p_{k}}\right)$

where $\theta>0$ is a real number and one defines $\delta_{k}$ which indicates the power of $\theta$ as follows:

$\left\{\begin{array}{l}\delta_{k}=2^{q-k}\left(\prod_{i=k+1}^{q}\left(\lambda_{i}-\frac{3}{2}\right)\right) \text { for } k=1, \ldots, q-1 \\ \delta_{q}=1 .\end{array}\right.$

Note that for any $k=1, \ldots, q-1$, one has

$\frac{\delta_{k}}{2}=\left(\lambda_{k+1}-\frac{3}{2}\right) \delta_{k+1}$.

Since $\lambda_{k} \geq 2$, one has $\left(\lambda_{k+1}-\frac{3}{2}\right) \geq \frac{1}{2}$ and therefore the $\delta_{k}$ 's constitute a decreasing sequence of positive real numbers, i.e.

$\delta_{1} \geq \delta_{2} \geq \cdots \geq \delta_{q}=1$.
One also defines the following sequence of scalar numbers for $k=1, \ldots, q$ and $i=1, \ldots, \lambda_{k}$ :

$\sigma_{i}^{k}=\sigma_{1}^{k}+(i-1) \delta_{k}$

$$
\text { with } \sigma_{1}^{k}=-\left(\lambda_{k}-1\right) \delta_{k}+\left(\lambda_{1}-1\right) \delta_{1}+\eta\left(1-\frac{1}{2^{k-1}}\right)
$$

where $0<\eta \leq 1$ can be chosen arbitrarily small.

It is easy to see that $\sigma_{i}^{k} \geq 0$, for $k=1, \ldots, q$ and $i=1, \ldots, \lambda_{k}$. Indeed, for $k=1$, one has

$$
\begin{aligned}
\sigma_{i}^{1} & =\sigma_{1}^{1}+(i-1) \delta_{1} \\
& =(i-1) \delta_{1} \\
& \geq 0 \quad \text { for } i \geq 1 \text { since } \delta_{1}>0 .
\end{aligned}
$$

For $k \geq 2$, one has

$$
\begin{aligned}
\sigma_{i}^{k} & =\left(\lambda_{1}-1\right) \delta_{1}-\left(\lambda_{k}-i\right) \delta_{k}+\eta\left(1-\frac{1}{2^{k-1}}\right) \\
& \geq\left(\lambda_{1}-1\right) \delta_{1}-\left(\lambda_{k}-1\right) \delta_{k} \\
& =\left(\lambda_{1}-1\right) \delta_{1}-\frac{\delta_{k-1}}{2}-\frac{\delta_{k}}{2} \text { according to (14) } \\
& \geq\left(\lambda_{1}-1\right) \delta_{1}-\frac{\delta_{1}}{2}-\frac{\delta_{1}}{2} \text { according to (15) } \\
& =\left(\lambda_{1}-2\right) \delta_{1} \geq 0 .
\end{aligned}
$$

Similarly to the $\Delta_{k}$ 's, one defines for $k=1, \ldots, q$ the diagonal matrices $\Lambda_{k}$ 's as follows:

$$
\begin{aligned}
\Lambda_{k}(\theta) & =\theta^{-\sigma_{1}^{k}} \Delta_{k}(\theta) \\
& =\operatorname{diag}\left(\frac{1}{\theta^{\sigma_{1}^{k}}} I_{p_{k}}, \frac{1}{\theta^{\sigma_{2}^{k}}} I_{p_{k}}, \ldots, \frac{1}{\theta^{\sigma_{\lambda_{k}}^{k}}} I_{p_{k}}\right) .
\end{aligned}
$$

Note that, according to the definition of the $\sigma_{i}^{k \prime}$ s given by (16), one has

$\sigma_{\lambda_{k}}^{k}=\left(\lambda_{1}-1\right) \delta_{1}+\eta\left(1-\frac{1}{2^{k-1}}\right)$

and therefore one has

$\sigma_{\lambda_{k}}^{k}-\sigma_{\lambda_{l}}^{l}=\eta\left(\frac{1}{2^{l-1}}-\frac{1}{2^{k-1}}\right) \quad$ for $k, l=1, \ldots, q$.

This means that whatever is the difference between $\lambda_{k}$ and $\lambda_{l}$, the difference between $\sigma_{\lambda_{k}}^{k}$ and $\sigma_{\lambda_{l}}^{l}$ (which are the powers of $\theta$ on the last rows of $\Lambda_{k}(\theta)$ and $\Lambda_{l}(\theta)$, respectively) can be made as small as desired by choosing $\eta$ small enough (very close to zero).

Now, taking into account the structures of $\Lambda_{k}(\theta), \Delta_{k}(\theta)$ and $A_{k}$, respectively, given by (19), (12) and (2), one can show that the following identities hold:

- $\Lambda_{k}(\theta) A_{k} \Lambda_{k}^{-1}(\theta)=\Delta_{k}(\theta) A_{k} \Delta_{k}^{-1}(\theta)=\theta^{\delta_{k}} A_{k}$

- $\theta^{-\sigma_{1}^{k}} C_{k} \Lambda_{k}^{-1}(\theta)=C_{k} \Delta_{k}^{-1}(\theta)=C_{k}$.

Another matrix that shall be used in the observer equation is $S_{k}$ and it is the unique solution of the algebraic Lyapunov equation

$$
S_{k}+A_{k}^{T} S_{k}+S_{k} A_{k}=C_{k}^{T} C_{k}
$$

where $A_{k}$ and $C_{k}$ are defined in (2) and (3), respectively. It can be shown that the explicit solution of (22) is symmetric positive definite and in particular, one has $[13,17]$

$$
\begin{aligned}
S_{k}^{-1} C_{k}^{T}= & \left(C_{\lambda_{k}}^{1} I_{p_{k}}, \ldots, C_{\lambda_{k}}^{\lambda_{k}} I_{p_{k}}\right)^{T} \\
& \text { where } C_{\lambda_{k}}^{i}=\frac{\lambda_{k} !}{i !\left(\lambda_{k}-i\right) !} \text { for } i=1, \ldots, \lambda_{k} .
\end{aligned}
$$


In what follows, one shall denote by $\lambda_{\min }^{k}$ and $\lambda_{\max }^{k}$ the smallest and largest eigenvalues of $S_{k}$, respectively. The variables $\lambda_{\min }$ and $\lambda_{\max }$ shall respectively denote the smallest and largest eigenvalues of $S$ defined as follows:

$S=\operatorname{diag}\left(S_{1}, S_{2}, \ldots, S_{q}\right)$.

Before ending this section, one shall give a technical lemma needed in the proof of our main result. This lemma allows to provide a sequence of reals that reflects in some sense the interconnections between the subsystem nonlinearities.

Lemma 2.1. Let

$\chi_{l, j}^{k, i}= \begin{cases}0 & \text { if } \frac{\partial \varphi_{i}^{k}}{\partial x_{j}^{l}}(u, x) \equiv 0 \\ 1 & \text { otherwise }\end{cases}$

for $k, l=1, \ldots, q, i=1, \ldots, \lambda_{k}$ and $j=2, \ldots, \lambda_{l}$. Then, the sequence of real numbers $\sigma_{i}^{k}$ defined by (16) is such that

if $\chi_{l, j}^{k, i}=1$ then $\sigma_{j}^{l}-\sigma_{i}^{k}-\frac{\delta_{l}}{2}-\frac{\delta_{k}}{2} \leq-\frac{\eta}{2^{q}}$.

Proof of Lemma 2.1. This shall be done using the following two facts. First, according to the state dependence given by (4) and (5), the case where $\chi_{l, j}^{k, i}=1$ for $k, l \in\{1, \ldots, q\}$ and $j \in\left\{2, \ldots, \lambda_{l}\right\}$ occurs if and only if one of the following three situations is met.

- $k>l$ and $i$ takes any value in $\left\{1, \ldots, \lambda_{k}\right\}$; this traduces the triangular like structure of the overall system.

- $k=l$ and $i \in\left\{1, \ldots, \lambda_{k}\right\}$ with $i \geq j$; this is related to the triangular structure of each subsystem with respect to its variables.

- $k<l$ and $i=\lambda_{k}$; this allows the last layer of each subsystem to depend on all state variables.

Second, it follows from (16) that

$$
\begin{aligned}
\sigma_{j}^{l}- & \sigma_{i}^{k}-\frac{\delta_{l}}{2}-\frac{\delta_{k}}{2} \\
= & \sigma_{1}^{l}+(j-1) \delta_{l}-\sigma_{1}^{k}-(i-1) \delta_{k}-\frac{\delta_{l}}{2}-\frac{\delta_{k}}{2} \\
= & -\left(\lambda_{l}-1\right) \delta_{l}+(j-1) \delta_{l}+\left(\lambda_{k}-1\right) \delta_{k}-(i-1) \delta_{k} \\
& +\eta\left(\frac{1}{2^{k-1}}-\frac{1}{2^{l-1}}\right)-\frac{\delta_{l}}{2}-\frac{\delta_{k}}{2} \\
= & \left(\lambda_{k}-i-\frac{1}{2}\right) \delta_{k}+\left(j-\lambda_{l}-\frac{1}{2}\right) \delta_{l}+\eta\left(\frac{1}{2^{k-1}}-\frac{1}{2^{l-1}}\right) .
\end{aligned}
$$

Let us now check condition (26) by considering the three cases listed above using (27).

- The case $k>l$ and $i$ takes any value in $\left\{1, \ldots, \lambda_{k}\right\}$. Let us first note that since $k>l$, one has $k \geq 2$ and hence equality (27) becomes

$$
\begin{aligned}
\sigma_{j}^{l} & -\sigma_{i}^{k}-\frac{\delta_{l}}{2}-\frac{\delta_{k}}{2} \\
& \leq\left(\lambda_{k}-\frac{3}{2}\right) \delta_{k}-\frac{\delta_{l}}{2}+\eta\left(\frac{1}{2^{k-1}}-\frac{1}{2^{l-1}}\right) \\
& =\frac{\delta_{k-1}}{2}-\frac{\delta_{l}}{2}+\eta\left(\frac{1}{2^{k-1}}-\frac{1}{2^{l-1}}\right) \text { according to (14) } \\
& \leq \eta\left(\frac{1}{2^{k-1}}-\frac{1}{2^{l-1}}\right) \text { according to (15) } \\
& =\frac{\eta}{2^{k-1}}\left(1-2^{k-l}\right) \leq-\frac{\eta}{2^{k-1}} \leq-\frac{\eta}{2^{q-1}} \leq-\frac{\eta}{2^{q}}
\end{aligned}
$$

- The case $k=l$ and $i \in\left\{1, \ldots, \lambda_{k}\right\}$ with $i \geq j$. Equality (27) becomes

$$
\sigma_{j}^{l}-\sigma_{i}^{k}-\frac{\delta_{l}}{2}-\frac{\delta_{k}}{2}=(j-i-1) \delta_{k} \leq-\delta_{k} \leq-1 \leq-\frac{\eta}{2^{q}} .
$$

- The case $k<l$ and $i=\lambda_{k}$. Equality (27) becomes

$$
\begin{aligned}
\sigma_{j}^{l} & -\sigma_{i}^{k}-\frac{\delta_{l}}{2}-\frac{\delta_{k}}{2} \\
& =-\frac{\delta_{k}}{2}+\left(j-\lambda_{l}-\frac{1}{2}\right) \delta_{l}+\eta\left(\frac{1}{2^{k-1}}-\frac{1}{2^{l-1}}\right) \\
& \leq-\frac{\delta_{k}}{2}-\frac{\delta_{l}}{2}+\eta\left(\frac{1}{2^{k-1}}-\frac{1}{2^{l-1}}\right) \\
& \leq-1+\frac{\eta}{2^{k-1}}\left(1-2^{k-l}\right) \quad \text { since } \delta_{k} \geq \delta_{l} \geq 1 \\
& \leq-1+\frac{\eta}{2^{k-1}}\left(1-\frac{1}{2}\right) \quad \text { since } l-k \geq 1 \\
& \leq-1+\frac{\eta}{2^{k}} \\
& \leq-1+\frac{\eta}{2} \\
& \leq-\frac{\eta}{2} \quad \text { since } \eta \leq 1 \\
& \leq-\frac{\eta}{2^{q}} .
\end{aligned}
$$

This ends the proof of the lemma.

Remark 2.1. Lemma 2.1 is the main key feature for the next developments, namely, for proving the convergence of the observer that we shall propose for system (1). Indeed, it is worth mentioning that under the classical Lipschitz condition on the nonlinearities, the main issue consists in proving that the norm of each $\left(p_{k} \times p_{l}\right)$ block entry of the following $n_{k} \times n_{l}$ matrix: $\theta^{-\frac{\delta_{l}}{2}-\frac{\delta_{k}}{2}} \Lambda_{k}(\theta) \frac{\partial \varphi^{k}}{\partial x^{l}}(u, x) \Lambda_{l}^{-1}(\theta)$, where $\Lambda_{k}$ and $\Lambda_{l}$ are defined by (19), can be made arbitrarily small for relatively high values of $\theta(\geq 1)$. Taking into account the diagonal structure of the matrices $\Lambda_{k}$ and $\Lambda_{l}$, it is easy to see that the block matrix entry located at the $i$ th row and the $j$ th column is either zero (if $\chi_{l, j}^{k, i}=0$ ), or depends only on $\theta$ through a multiplying term equal to $\theta^{-\sigma_{i}^{k}+\sigma_{j}^{l}-\frac{\delta_{l}}{2}-\frac{\delta_{k}}{2}}$ Thanks to Lemma 2.1 , such a term can be made arbitrarily small for $\theta \geq 1$ since the power of $\theta$ is negative.

\subsection{Discussion of the considered class of systems}

The structure of system (1) exhibits three novelties with respect to available contributions related to high gain observer design (see for instance [19] and the reference list therein).

(i) The output of each block may be a vector and is not necessary a single signal.

(ii) The last differential equation in each block may depend on all the state and therefore the overall system does not assume a triangular structure.

(iii) The last differential equation in each block may involve some uncertainties.

In order to motivate item (i), consider the following example:

$\left\{\begin{array}{l}\dot{x}_{1}=x_{3} \\ \dot{x}_{3}=x_{5}+u\left(x_{3}+x_{4}\right) \\ \dot{x}_{5}=-u\left(x_{5}+x_{6}\right)+u x_{8} \\ y_{1}=x_{1}\end{array}\right.$ 


$$
\begin{aligned}
& \left\{\begin{array}{l}
\dot{x}_{2}=x_{4} \\
\dot{x}_{4}=x_{6}+u\left(x_{3}-x_{4}\right) \\
\dot{x}_{6}=-u\left(x_{5}-x_{6}\right) \\
y_{2}=x_{2}
\end{array}\right. \\
& \left\{\begin{array}{l}
\dot{x}_{7}=x_{8}+u\left(x_{5}+x_{6}\right) \\
\dot{x}_{8}=u\left(x_{5}+x_{6}\right) \\
y_{3}=x_{7} .
\end{array}\right.
\end{aligned}
$$

It is easy to see that the overall system composed of the three blocks (28)-(30) is not in the form (1) since the second equation of the second block does depend on the second component of the first block and vice versa. Let us perform a simple rearrangement by grouping together the first two outputs $x_{1}$ and $x_{2}$. Indeed, set $x_{1}^{1}=\left(\begin{array}{l}x_{1,1}^{1}=x_{1} \\ x_{1,2}^{1}=x_{2}\end{array}\right), x_{2}^{1}=\left(\begin{array}{l}x_{2,1}^{1}=x_{3} \\ x_{2,2}^{1}=x_{4}\end{array}\right), x_{3}^{1}=\left(\begin{array}{l}x_{3,1}^{1}=x_{5} \\ x_{3,2}^{1}=x_{6}\end{array}\right), x^{1}=$ $\left(\begin{array}{l}x^{1} \\ x^{2} \\ x^{3}\end{array}\right), x^{2}=\left(\begin{array}{l}x_{1}^{2}=x_{7} \\ x_{2}^{2}=x_{8}\end{array}\right)$. Using these notations, system (28)-(30) can be written in the following form

$$
\begin{aligned}
& \left\{\begin{array}{l}
\dot{x}_{1}^{1}=x_{2}^{1} \\
\dot{x}_{2}^{1}=x_{3}^{1}+u\left(\begin{array}{cc}
1 & 1 \\
1 & -1
\end{array}\right) x_{2}^{1} \\
\dot{x}_{3}^{1}=-u\left(\begin{array}{cc}
1 & 1 \\
1 & -1
\end{array}\right) x_{3}^{1}+\left(\begin{array}{l}
0 \\
u
\end{array}\right) x_{2}^{2} \\
\bar{y}_{1}=x^{1}=\left(\begin{array}{l}
y_{1} \\
y_{2}
\end{array}\right)
\end{array}\right. \\
& \left\{\begin{array}{l}
\dot{x}_{1}^{2}=x_{2}^{2}+u\left(x_{3,1}^{1}+x_{3,2}^{1}\right) \\
\dot{x}_{2}^{2}=u\left(x_{3,1}^{1}+x_{3,2}^{1}\right) \\
\bar{y}_{2}=x_{1}^{2}
\end{array}\right.
\end{aligned}
$$

which is clearly in the form (1) with $q=2$, the overall output $y=\left(\begin{array}{l}\bar{y}_{1} \\ \bar{y}_{2}\end{array}\right), p_{1}=2$ and $p_{2}=1$. In item (ii), the non-triangular structure of system (1) is put forward. Such a structure results from the dependence of the last equation of each block on the whole state. Such a feature constitutes the main novelty in system (1) with respect to existing works related to the observer design, e.g. the class of systems considered in [19] is similar to system (1) but it assumes a triangular structure without uncertainties. The techniques used in this paper to prove the main result are similar to those usually adopted when designing high gain observers (see $[19,21]$ for instance). In these works, the expressions of the variables $\delta_{k}$ and $\sigma_{i}^{k}$ given by (13) and (16) differ from those given in this paper. Indeed, in [19], these variables are defined as follows:

$$
\begin{aligned}
& \delta_{k}=\prod_{i=1}^{k-1} \lambda_{k} \quad \text { for } k=2, \ldots, q \text { with } \delta_{1}=1 \\
& \sigma_{i}^{k}=i \delta_{k} \quad k=1, \ldots, q \text { and } i=1, \ldots, \lambda_{k} .
\end{aligned}
$$

With $\delta_{k}$ and $\sigma_{i}^{k}$, respectively, defined as in (33) and (34), the inequality (26) given in Lemma 2.1 is by no means fully checked. More precisely, one cannot check this inequality in the three cases described in the proof of lemma but it can only be proved in the first two cases, i.e. $k>l$ and $k=l$. For the third case $(k<l)$ which induces the non-triangular structure of the overall system, inequality (26) does not hold. Let us check all these statements by considering the three cases of Lemma 2.1. First of all, using the expressions of the variables $\delta_{k}$ 's and $\sigma_{i}^{k}$ 's given by (33) and (34), respectively, one has:

$\sigma_{j}^{l}-\sigma_{i}^{k}-\frac{\delta_{l}}{2}-\frac{\delta_{k}}{2}=j \delta_{l}-i \delta_{k}-\frac{\delta_{l}}{2}-\frac{\delta_{k}}{2}$
- $k>l$ : one has

$$
\begin{aligned}
\sigma_{j}^{l} & -\sigma_{i}^{k}-\frac{\delta_{l}}{2}-\frac{\delta_{k}}{2} \\
& \leq \lambda_{l} \delta_{l}-\delta_{k}-\frac{\delta_{l}}{2}-\frac{\delta_{k}}{2} \\
& =\delta_{l+1}-\delta_{k}-\frac{\delta_{l}}{2}-\frac{\delta_{k}}{2} \text { according to (33) } \\
& \leq-\frac{\delta_{l}}{2}-\frac{\delta_{k}}{2} \text { since the } \delta_{k} \text { 's given by (33) } \\
& \text { define a non-decreasing sequence } \\
< & 0 .
\end{aligned}
$$

- $k=l$ and $j \leq i$ : one has

$$
\sigma_{j}^{l}-\sigma_{i}^{k}-\frac{\delta_{l}}{2}-\frac{\delta_{k}}{2} \leq(j-i-1) \delta_{k} \leq-\delta_{k}<0
$$

- $k<l$ and $i=\lambda_{k}$ : in this case, one shall show that inequality (26) cannot be satisfied even with $j=2$. This means that the last layer of each block must have the same triangular structure as the other layers of the block. Indeed, one has:

$$
\begin{aligned}
\sigma_{j}^{l}-\sigma_{i}^{k}-\frac{\delta_{l}}{2}-\frac{\delta_{k}}{2} & =\left(j-\frac{1}{2}\right) \delta_{l}-\left(\lambda_{k}+\frac{1}{2}\right) \delta_{k} \\
& =\left(j-\frac{1}{2}\right) \delta_{l}-\delta_{k+1}-\frac{\delta_{k}}{2} \\
& \geq\left(j-\frac{1}{2}\right) \delta_{l}-\delta_{l}-\frac{\delta_{k}}{2} \\
& \geq\left(2-\frac{1}{2}\right) \delta_{l}-\delta_{l}-\frac{\delta_{k}}{2} \\
& =\frac{\delta_{l}}{2}-\frac{\delta_{k}}{2}>0 .
\end{aligned}
$$

In item (iii), one focuses on the system uncertainties. The consideration of the uncertainties in the last equation of each block gives rise to difficulties in the observer design similar to those described when dealing with item (ii). It is well known that in the case of a single block, one can design a high observer which allows to obtain an estimation error with an ultimate bound that can be made as small as desired by choosing relatively high values for the design parameter $\theta$. For system (1), there are two additional difficulties that should be addressed. The first is related to the nontriangular dependence assumed in the last equation where each uncertainty appears. The tools used to cope with problem have been described above. The second major problem deals with the fact that the $\lambda_{k}$ 's are not necessarily equal for $k=1, \ldots, q$. This difficulty was surmounted thanks to property (20). More details are of course given in the proof of our main result in the next section.

\section{Observer design}

As generally assumed in the high gain observer design [26,13, $27,21,20,28]$, one considers the following Lipschitz assumption.

Assumption 1. $\varphi(u, x)$ is a globally Lipschitz nonlinear function with respect to $x$ uniformly in $u$.

Remark 3.1. Assumption 1 would be too restrictive since the Lipschitz conditions are in general locally satisfied. However, these conditions can be omitted in the case where the system state trajectory lies in a bounded set $\Omega$. In such a case, one can extend the nonlinearities $\varphi(u, x)$ into $\tilde{\varphi}(u, x)$ in such a way that the restriction of $\widetilde{\varphi}(u, x)$ coincides with $\varphi(u, x)$ on $\Omega$ and $\widetilde{\varphi}(u, x)$ becomes global Lipschitz on the whole state $\mathbb{R}^{n}$. Prolongation techniques were 
used in $[26,13]$ and have been recently detailed in $[29,19]$. As the description of these techniques is not the subject of this work, one shall assume that the prolongations are achieved if necessary and system (1) will be considered on $\mathbb{R}^{n}$.

Bearing in mind the high gain design concept, a candidate observer for system (1) is described by the following dynamical system

$\dot{\hat{x}}^{k}=A_{k} \hat{x}^{k}+\varphi^{k}(u, \underline{\hat{x}})-\theta^{\delta_{k}} \Delta_{k}^{-1}(\theta) S_{k}^{-1} C_{k}^{T} C_{k} \mathrm{e}^{k}$

for $k=1, \ldots, q$

where $u$ and $y$ are respectively the inputs and outputs of system (1), $\hat{x} \in \mathbb{R}^{n}$ denotes the state estimate given by

$$
\begin{aligned}
& \hat{x}=\left(\begin{array}{c}
\hat{x}^{1} \\
\hat{x}^{2} \\
\vdots \\
\hat{x}^{q}
\end{array}\right) \in \mathbb{R}^{n} \quad \text { with } \hat{x}^{k}=\left(\begin{array}{c}
\hat{x}_{1}^{k} \\
\hat{x}_{2}^{k} \\
\vdots \\
\hat{x}_{\lambda_{k}}^{k}
\end{array}\right) \in \mathbb{R}^{n_{k}} \\
& \text { and } \hat{x}_{i}^{k}=\left(\begin{array}{c}
\hat{x}_{i, 1}^{k} \\
\vdots \\
\hat{x}_{i, p_{k}}^{k}
\end{array}\right) \in \mathbb{R}^{p_{k}}
\end{aligned}
$$

where $\hat{x}_{i, j}^{k} \in \mathbb{R}$, for $k=1, \ldots, q, i=1, \ldots, \lambda_{k}, j=1, \ldots, p_{k}$ and hence $\sum_{k=1}^{q} n_{k}=n, \hat{x}^{k} \in \mathbb{R}^{n_{k}}$ denotes a state estimate up to an output injection, namely,

$\underline{\hat{x}}_{i}^{k}= \begin{cases}x_{1}^{k} & \text { for } i=1 \\ \hat{x}_{i}^{k} & \text { for } i=2, \ldots, \lambda_{k}\end{cases}$

and $\mathrm{e}^{k} \in \mathbb{R}^{n_{k}}$ is the $i$ th component of the observation error vector and is defined by

$\mathrm{e}^{k}=\hat{x}^{k}-x^{k}$.

The following result provides the fundamental properties of the observer.

Theorem 3.1. Assume that system (1) satisfies Assumption 1, then $\forall M>0 ; \exists \theta_{0}>0 ; \forall \theta>\theta_{0} ; \exists \lambda_{\theta}>0 ; \exists \mu_{\theta}>0 ; \exists \alpha_{\theta}>0$ such that for $k \in\{1, \ldots, q\}$

$\left\|\hat{x}^{k}(t)-x^{k}(t)\right\| \leq \lambda_{\theta} \mathrm{e}^{-\mu_{\theta} t}\|\hat{x}(0)-x(0)\|+\alpha_{\theta} \beta$

for every admissible control $u$ s.t. $\|u\|_{\infty} \leq M$, where $\beta$ is the upper bound of $\left\|\varepsilon_{k}\right\|$ given in (6). Moreover, $\lambda_{\theta}$ is a polynomial in $\theta, \lim _{\theta \rightarrow \infty} \mu_{\theta}=+\infty$ and $\lim _{\theta \rightarrow \infty} \alpha_{\theta}=0$.

Proof of Theorem 3.1. Set the estimation error $e(t)=\hat{x}(t)-x(t)$ and let $\mathrm{e}^{k}(t)$ be the $k$ th subcomponent of $e(t)$. For writing convenience and as long as there is no ambiguity, one shall omit the time $t$ for each variable. One has:

$\dot{\mathrm{e}}^{k}=A_{k} \mathrm{e}^{k}+\varphi^{k}(u, \underline{\hat{x}})-\varphi^{k}(u, x)-\theta^{\delta_{k}} \Delta_{k}^{-1}(\theta) S_{k}^{-1} C_{k}^{T} C_{k} \mathrm{e}^{k}-\bar{\varepsilon}^{k}$

where $u$ is an admissible control such that $\|u\|_{\infty} \leq M$ where $M>0$ is a given constant.

For $k=1, \ldots, q$, set

$\overline{\mathrm{e}}^{k}=\Lambda_{k}(\theta) \mathrm{e}^{k}$

where $\Lambda_{k}(\theta)$ is given by (19).

From Eq. (36) and using identities (21) and (37), one gets

$$
\begin{aligned}
\dot{\overline{\mathrm{e}}}^{k}= & \Lambda_{k}(\theta) A_{k} \Lambda_{k}(\theta)^{-1} \overline{\mathrm{e}}^{k}+\Lambda_{k}(\theta)\left(\varphi^{k}(u, \underline{\hat{x}})-\varphi^{k}(u, x)\right) \\
& -\Lambda_{k}(\theta) \bar{\varepsilon}^{k}-\theta^{\delta_{k}} \Lambda_{k}(\theta) \Delta_{k}^{-1}(\theta) S_{k}^{-1} C_{k}^{T} C_{k} \Lambda_{k}(\theta)^{-1} \overline{\mathrm{e}}^{k} \\
= & \theta^{\delta_{k}} A_{k} \overline{\mathrm{e}}^{k}-\theta^{\delta_{k}} S_{k}^{-1} C_{k}^{T} C_{k} \overline{\mathrm{e}}^{k}+\Lambda_{k}(\theta)\left(\varphi^{k}(u, \underline{\hat{x}})\right. \\
& \left.-\varphi^{k}(u, x)\right)-\Lambda_{k}(\theta) \bar{\varepsilon}^{k} .
\end{aligned}
$$

Set

$V_{k}\left(\overline{\mathrm{e}}^{k}\right)=\overline{\mathrm{e}}^{k^{T}} S_{k} \overline{\mathrm{e}}^{k}$

where $S_{k}$ is given by (22) and let $V(\bar{e})=\sum_{k=1}^{q} V_{k}\left(\overline{\mathrm{e}}^{k}\right)$ be the candidate Lyapunov function.

Note that one has:

$V(\bar{e}) \leq \lambda_{\max }\|\bar{e}\|^{2}$.

And recall that $\lambda_{\max }$ denotes the largest eigenvalue of the matrix $S$ given by (24).

In what follows, the value of the design parameter $\theta$ will assumed to be greater than or equal to 1 , i.e. $\theta \geq 1$.

The time derivative of the function $V_{k}$ is given by

$$
\begin{aligned}
\dot{V}_{k}= & 2 \overline{\mathrm{e}}^{-k^{T}} S_{k} \dot{\mathrm{e}}^{k} \\
= & 2 \theta^{\delta k} \mathrm{e}^{-k^{T}} S_{k} A_{k} \overline{\mathrm{e}}^{k}-2 \theta^{\delta k} \overline{\mathrm{e}}^{k^{T}} C_{k}^{T} C_{k} \overline{\mathrm{e}}^{k} \\
& +2 \overline{\mathrm{e}}^{k^{T}} S_{k} \Lambda_{k}(\theta)\left(\varphi^{k}(u, \underline{\hat{x}})-\varphi^{k}(u, x)\right)-2 \overline{\mathrm{e}}^{k^{T}} S_{k} \Lambda_{k}(\theta) \bar{\varepsilon}^{k} .
\end{aligned}
$$

Using the algebraic Lyapunov equation (22) yields

$$
\begin{aligned}
\dot{V}_{k}= & -\theta^{\delta_{k}} \overline{\mathrm{e}}^{k^{T}} S_{k} \overline{\mathrm{e}}^{k}-\theta^{\delta_{k}} \mathrm{e}^{-k^{T}} C_{k}^{T} C_{k} \overline{\mathrm{e}}^{k} \\
& +2 \mathrm{e}^{k^{T}} S_{k} \Lambda_{k}(\theta)\left(\varphi^{k}(u, \underline{\hat{x}})-\varphi^{k}(u, x)\right)-2 \mathrm{e}^{k^{T}} S_{k} \Lambda_{k}(\theta) \bar{\varepsilon}^{k} \\
\leq & -\theta^{\delta_{k}} \overline{\mathrm{e}}^{-k^{T}} S_{k} \overline{\mathrm{e}}^{k}+2 \mathrm{e}^{k^{T}} S_{k} \Lambda_{k}(\theta)\left(\varphi^{k}(u, \underline{\hat{x}})\right. \\
& \left.-\varphi^{k}(u, x)\right)-2 \overline{\mathrm{e}}^{k^{T}} S_{k} \Lambda_{k}(\theta) \bar{\varepsilon}^{k} \\
\leq & -\theta^{\delta_{k}} V_{k}+2\left\|S_{k} \overline{\mathrm{e}}^{k}\right\| \| \Lambda_{k}(\theta)\left(\varphi^{k}(u, \underline{\hat{x}})\right. \\
& \left.-\varphi^{k}(u, x)\right)\|+2\| S_{k} \overline{\mathrm{e}}^{k}\|\| \Lambda_{k}(\theta) \bar{\varepsilon}^{k} \| \\
\leq & -\theta^{\delta_{k}} V_{k}+2 \sqrt{\lambda_{\max }^{k}} \sqrt{V_{k}} \sum_{i=1}^{\lambda_{k}} \frac{1}{\theta^{\sigma_{i}^{k}}}\left\|\left(\varphi_{i}^{k}(u, \underline{\hat{x}})-\varphi_{i}^{k}(u, x)\right)\right\| \\
& +2 \sqrt{\lambda_{\max }^{k}} \sqrt{V_{k}} \frac{\beta}{\theta^{\sigma_{1}^{k}+\left(\lambda_{k}-1\right) \delta_{k}}}
\end{aligned}
$$

where $\sigma_{i}^{k}=\sigma_{1}^{k}+(i-1) \delta_{k}$ is as given in (16).

In addition, taking into account Assumption 1, i.e. the boundedness of $\frac{\partial \varphi}{\partial x}$, one has

$$
\begin{aligned}
\dot{V}_{k} \leq & -\theta^{\delta_{k}} V_{k}+2 \rho_{k} \sqrt{\lambda_{\max }^{k}} \sqrt{V_{k}} \sum_{i=1}^{\lambda_{k}} \sum_{l=1}^{q} \sum_{j=2}^{\lambda_{l}} \chi_{l, j}^{k, i} \theta^{-\sigma_{i}^{k}}\left\|\mathrm{e}_{j}^{l}\right\| \\
& +2 \sqrt{\lambda_{\max }^{k}} \sqrt{V_{k}} \frac{\beta}{\theta^{\sigma_{1}^{k}+\left(\lambda_{k}-1\right) \delta_{k}}}
\end{aligned}
$$

where $\rho_{k}=\sup \left\{\left\|\frac{\partial \varphi_{i}^{k}}{\partial x_{j}^{l}}(u, x)\right\| / x \in \mathbb{R}^{n}\right.$ and $\left.\|u\|_{\infty} \leq M\right\}$ and the $\chi_{l, j}^{k, i}$ s have the same definition as in Lemma 2.1. Such an inequality may be rewritten as follows

$$
\begin{aligned}
\dot{V}_{k} \leq & -\theta^{\delta_{k}} V_{k}+2 \rho_{k} \sqrt{\lambda_{\max }^{k}} \sqrt{V_{k}} \sum_{i=1}^{\lambda_{k}} \sum_{l=1}^{q} \sum_{j=2}^{\lambda_{l}} \chi_{l, j}^{k, i} \theta^{\sigma_{j}^{l}-\sigma_{i}^{k}}\left\|\bar{e}_{j}^{l}\right\| \\
& +2 \sqrt{\lambda_{\max }^{k}} \sqrt{V_{k}} \frac{\beta}{\theta^{\sigma_{1}^{k}+\left(\lambda_{k}-1\right) \delta_{k}}}
\end{aligned}
$$

where $\sigma_{j}^{l}$ is defined as in (16), i.e. $\sigma_{j}^{l}=\sigma_{1}^{l}+(j-1) \delta_{l}$.

Finally, one obtains

$$
\begin{aligned}
\dot{V}_{k} \leq & -\theta^{\delta_{k}} V_{k}+2 \rho_{k} \mu_{S} \sqrt{V_{k}} \sum_{i=1}^{\lambda_{k}} \sum_{l=1}^{q} \sum_{j=2}^{\lambda_{l}} \chi_{l, j}^{k, i} \theta^{\sigma_{j}^{l}-\sigma_{i}^{k}} \sqrt{V_{l}} \\
& +2 \sqrt{\lambda_{\max }^{k}} \sqrt{V_{k}} \frac{\beta}{\theta^{\sigma_{1}^{k}+\left(\lambda_{k}-1\right) \delta_{k}}}
\end{aligned}
$$




$$
\begin{aligned}
= & -\theta^{\delta_{k}} V_{k}+2 \rho_{k} \mu_{S} \sqrt{\theta^{\delta_{k}} V_{k}} \sum_{i=1}^{\lambda_{k}} \sum_{l=1}^{q} \sum_{j=2}^{\lambda_{l}} \chi_{l, j}^{k, i} \\
& \times \theta^{\sigma_{j}^{l}-\sigma_{i}^{k}-\frac{\delta_{l}}{2}-\frac{\delta_{k}}{2}} \sqrt{\theta^{\delta_{l}} V_{l}}+2 \sqrt{\lambda_{\max }^{k}} \sqrt{V_{k}} \frac{\beta}{\theta^{\sigma_{1}^{k}+\left(\lambda_{k}-1\right) \delta_{k}}}
\end{aligned}
$$

where $\mu_{S}=\sqrt{\frac{\lambda_{\max }}{\lambda_{\min }}}$.

Moreover, according to Lemma 2.1, one has

$$
\begin{aligned}
\sigma_{j}^{l}-\sigma_{i}^{k}-\frac{\delta_{l}}{2}-\frac{\delta_{k}}{2} & \leq-\frac{\eta}{2^{q}} \\
\sigma_{1}^{k}+\left(\lambda_{k}-1\right) \delta_{k} & =\left(\lambda_{1}-1\right) \delta_{1}+\eta\left(1-\frac{1}{2^{k-1}}\right) \\
& \geq\left(\lambda_{1}-1\right) \delta_{1}
\end{aligned}
$$

and taking into account (41) and (42), one has

$$
\begin{aligned}
\dot{V}_{k} \leq & -\theta^{\delta_{k}} V_{k}+2 \rho_{k} \mu_{S} \theta^{-\frac{\eta}{2^{q}}} \sqrt{\theta^{\delta_{k}} V_{k}} \sum_{i=1}^{\lambda_{k}} \sum_{l=1}^{q} \sum_{j=2}^{\lambda_{l}} \sqrt{\theta^{\delta_{l}} V_{l}} \\
& +2 \sqrt{\lambda_{\max }} \sqrt{V_{k}} \frac{\beta}{\theta^{\left(\lambda_{1}-1\right) \delta_{1}}} .
\end{aligned}
$$

Moreover, let

$V_{k}^{*}=\theta^{\delta_{k}} V_{k} \quad$ and $\quad V^{*}=\sum_{k=1}^{q} V_{k}^{*}$

it follows from (15) that

$V^{\star} \geq \theta^{\delta_{q}} V=\theta V$.

And inequality (43) becomes

$$
\begin{aligned}
\dot{V}_{k} \leq & -V_{k}^{\star}+2 \rho_{k} \mu_{S} \theta^{-\frac{\eta}{2^{q}}} \sqrt{V_{k}^{\star}} \sum_{i=1}^{\lambda_{k}} \sum_{l=1}^{q} \sum_{j=2}^{\lambda_{l}} \sqrt{V_{l}^{\star}} \\
& +2 \sqrt{\lambda_{\max }} \sqrt{V_{k}} \frac{\beta}{\theta^{\left(\lambda_{1}-1\right) \delta_{1}}} \\
= & -V_{k}^{\star}+2 \lambda_{k} \rho_{k} \mu_{S} \theta^{-\frac{\eta}{2^{q}}} \sqrt{V_{k}^{\star}} \sum_{l=1}^{q} \sum_{j=2}^{\lambda_{l}} \sqrt{V_{l}^{\star}} \\
& +2 \sqrt{\lambda_{\max }} \sqrt{V_{k}} \frac{\beta}{\theta^{\left(\lambda_{1}-1\right) \delta_{1}}} \\
\leq & -V_{k}^{\star}+2 \lambda_{k} \rho_{k} \mu_{S} \theta^{-\frac{\eta}{2^{q}}} \sqrt{V_{k}^{\star}} \sum_{l=1}^{q} \sum_{j=2}^{\lambda_{l}} \sqrt{V^{\star}} \\
& +2 \sqrt{\lambda_{\max }} \sqrt{V} \frac{\beta}{\theta^{\left(\lambda_{1}-1\right) \delta_{1}}} \\
\leq & -V_{k}^{\star}+2 n \lambda_{k} \rho_{k} \mu_{S} \theta^{-\frac{\eta}{2^{q}}} \sqrt{V_{k}^{\star}} \sqrt{V^{\star}} \\
& +2 \sqrt{\lambda_{\max }} \sqrt{V} \frac{\beta}{\theta^{\left(\lambda_{1}-1\right) \delta_{1}}} \\
\leq & -V_{k}^{\star}+2 n \lambda_{k} \rho_{k} \mu_{S} \theta^{-\frac{\eta}{2^{q}}} V^{\star} \\
& +2 \sqrt{\lambda_{\max }} \sqrt{V} \frac{\beta}{\theta^{\left(\lambda_{1}-1\right) \delta_{1}}} .
\end{aligned}
$$

Hence,

$$
\begin{aligned}
\dot{V} & \leq-V^{\star}+2 n^{2} \rho \mu_{S} \theta^{-\frac{\eta}{2^{q}}} V^{\star}+2 q \sqrt{\lambda_{\max }} \sqrt{V} \frac{\beta}{\theta^{\left(\lambda_{1}-1\right) \delta_{1}}} \\
& =-\left(1-2 n^{2} \rho \mu_{S} \theta^{-\frac{\eta}{2^{q}}}\right) V^{\star}+2 q \sqrt{\lambda_{\max }} \sqrt{V} \frac{\beta}{\theta^{\left(\lambda_{1}-1\right) \delta_{1}}}
\end{aligned}
$$

where $\rho=\max \left\{\rho_{k}\right.$ for $\left.1 \leq k \leq q\right\}$.
Furthermore, using (45) for $\theta$ high enough such that

$$
\left(1-2 n^{2} \rho \mu_{S} \theta^{-\frac{\eta}{2^{q}}}\right)>0
$$

yields

$\dot{V} \leq-\theta\left(1-2 n^{2} \rho \mu_{S} \theta^{-\frac{\eta}{2^{q}}}\right) V+2 q \sqrt{\lambda_{\max }} \sqrt{V} \frac{\beta}{\theta^{\left(\lambda_{1}-1\right) \delta_{1}}}$.

This leads to

$$
\begin{aligned}
\sqrt{V(\bar{e}(t))} \leq & \exp \left(-\frac{\theta\left(1-2 n^{2} \rho \mu_{S} \theta^{-\frac{\eta}{2^{q}}}\right)}{2} t\right) \sqrt{V(\bar{e}(0))} \\
& +\frac{2 q \sqrt{\lambda_{\max }} \beta}{\left(1-2 n^{2} \rho \mu_{S} \theta^{-\frac{\eta}{2^{q}}}\right) \theta^{\left(1+\left(\lambda_{1}-1\right) \delta_{1}\right)}}
\end{aligned}
$$

and therefore one obtains for $k=1, \ldots, q$

$$
\begin{aligned}
\sqrt{V_{k}\left(\bar{e}^{k}(t)\right)} \leq & \sqrt{V(\bar{e}(t))} \\
\leq & \exp \left(-\frac{\theta\left(1-2 n^{2} \rho \mu_{S} \theta^{-\frac{\eta}{2^{q}}}\right)}{2} t\right) \sqrt{V(\bar{e}(0))} \\
& +\frac{2 q \sqrt{\lambda_{\max }} \beta}{\left(1-2 n^{2} \rho \mu_{S} \theta^{-\frac{\eta}{2^{q}}}\right) \theta^{\left(1+\left(\lambda_{1}-1\right) \delta_{1}\right)}} .
\end{aligned}
$$

In other respects, it follows from Eqs. (37) and (39) that

$\frac{\sqrt{V_{k}\left(\overline{\mathrm{e}}^{k}\right)}}{\sqrt{\lambda_{\max }}} \leq \frac{\sqrt{V_{k}\left(\overline{\mathrm{e}}^{k}\right)}}{\sqrt{\lambda_{\max }^{k}}} \leq\left\|\overline{\mathrm{e}}^{k}\right\| \leq \frac{\sqrt{V_{k}\left(\overline{\mathrm{e}}^{k}\right)}}{\sqrt{\lambda_{\min }^{k}}} \leq \frac{\sqrt{V_{k}\left(\overline{\mathrm{e}}^{k}\right)}}{\sqrt{\lambda_{\min }}}$

and

$\left\|\overline{\mathrm{e}}^{k}\right\| \leq\left\|\mathrm{e}^{k}\right\| \leq \theta^{\sigma_{1}^{k}+\left(\lambda_{k}-1\right) \delta_{k}}\left\|\overline{\mathrm{e}}^{k}\right\|$.

Using (40) and according to (50), one has

$\sqrt{V(\bar{e})} \leq \sqrt{\lambda_{\max }}\|\bar{e}\| \leq \sqrt{\lambda_{\max }}\|e\|$

and combining (49) and (50), one has

$\left\|\mathrm{e}^{k}\right\| \leq \theta^{\sigma_{1}^{k}+\left(\lambda_{k}-1\right) \delta_{k}}\left\|\mathrm{e}^{-k}\right\| \leq \theta^{\sigma_{1}^{k}+\left(\lambda_{k}-1\right) \delta_{k}} \frac{\sqrt{V_{k}\left(\overline{\mathrm{e}}^{k}\right)}}{\sqrt{\lambda_{\min }}}$.

Finally, using inequalities (48) and (52), one obtains $\left\|\mathrm{e}^{k}(t)\right\| \leq \theta^{\sigma_{1}^{k}+\left(\lambda_{k}-1\right) \delta_{k}}$

$$
\begin{aligned}
& \times \frac{1}{\sqrt{\lambda_{\min }}} \exp \left(-\frac{\theta\left(1-2 n^{2} \rho \mu_{S} \theta^{-\frac{\eta}{2^{q}}}\right)}{2} t\right) \sqrt{V(\bar{e}(0))} \\
& +\frac{2 q \sqrt{\lambda_{\max }} \beta}{\sqrt{\lambda_{\min }}} \frac{\theta^{\sigma_{1}^{k}+\left(\lambda_{k}-1\right) \delta_{k}}}{\left(1-2 n^{2} \rho \mu_{S} \theta^{-\frac{\eta}{2 q}}\right) \theta^{\left(1+\left(\lambda_{1}-1\right) \delta_{1}\right)}} .
\end{aligned}
$$

And from (42) and (51), one gets

$$
\begin{aligned}
\left\|\mathrm{e}^{k}(t)\right\| \leq & \theta^{\left(\lambda_{1}-1\right) \delta_{1}+\eta\left(1-\frac{1}{2^{k-1}}\right)} \mu_{S} \\
& \times \exp \left(-\frac{\theta\left(1-2 n^{2} \rho \mu_{S} \theta^{-\frac{\eta}{2 q}}\right)}{2} t\right)\|e(0)\| \\
& +\frac{2 q \mu_{S} \theta^{\left(\lambda_{1}-1\right) \delta_{1}+\eta\left(1-\frac{1}{2^{k-1}}\right)}}{\left(1-2 n^{2} \rho \mu_{S} \theta^{-\frac{\eta}{2^{q}}}\right) \theta^{\left(1+\left(\lambda_{1}-1\right) \delta_{1}\right)}} \beta
\end{aligned}
$$



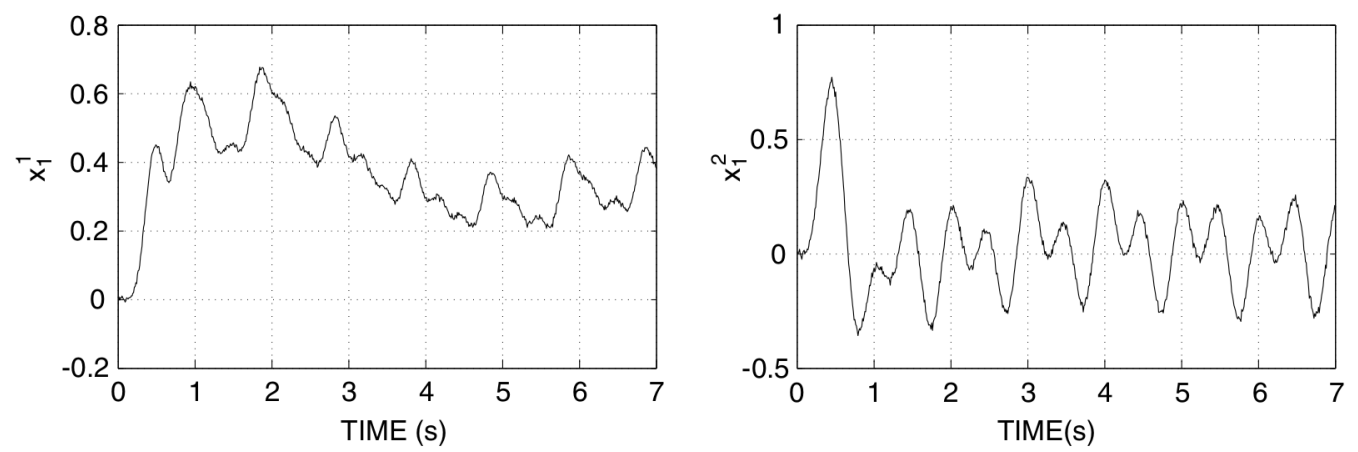

Fig. 1. Noisy measurements of $x_{1}^{1}$ and $x_{1}^{2}$.

$$
\begin{aligned}
\leq & \theta^{\left(\lambda_{1}-1\right) \delta_{1}+\eta} \mu_{S} \exp \left(-\frac{\theta\left(1-2 n^{2} \rho \mu_{S} \theta^{-\frac{\eta}{2^{q}}}\right)}{2} t\right)\|e(0)\| \\
& +\frac{2 q \mu_{S}}{\left(1-2 n^{2} \rho \mu_{S} \theta^{-\frac{\eta}{2^{q}}}\right) \theta^{1-\eta}} \beta
\end{aligned}
$$

This ends the proof of the theorem with the parameters $\theta_{0}, \lambda_{\theta}, \mu_{\theta}$ and $\beta_{\theta}$ given by

$$
\begin{aligned}
& \theta_{0}=\max \left(1,\left(2 n^{2} \rho \mu_{S}\right)^{\frac{2 q}{\eta}}\right) \\
& \lambda_{\theta}=\theta^{\left(\lambda_{1}-1\right) \delta_{1}+\eta} \mu_{S} \\
& \mu_{\theta}=\frac{\theta\left(1-2 n^{2} \rho \mu_{S} \theta^{-\frac{\eta}{2^{q}}}\right)}{2} \\
& \alpha_{\theta}=\frac{2 q \mu_{S}}{\theta^{1-\eta}\left(1-2 n^{2} \rho \mu_{S} \theta^{-\frac{\eta}{2^{q}}}\right)} .
\end{aligned}
$$

It is worth noticing that the observation error converges exponentially to zero in the absence of uncertainties and can be made as small as desired in the presence of uncertainties. Indeed, the observation error remains in a ball with a radius proportional to $\frac{1}{\theta^{1-\eta}}$. However, and as well known for high gain observers (see e.g. $[13,20]$ ), very large values of the design parameter $\theta$ are to be avoided in practice since the observer may become noise sensitive. Thus, the choice of $\theta$ is a compromise between fast convergence and sensitivity to noise and it is generally achieved using a trial and error strategy.

\section{An example}

In the following, simulation results are given to show the effectiveness of the proposed observer design using the following multi-output nonlinear system:

$$
\begin{aligned}
& \left\{\begin{array}{l}
\dot{x}_{1}^{1}=x_{2}^{1}-x_{1}^{1}+x_{1}^{2} u \\
\dot{x}_{2}^{1}=x_{3}^{1}-x_{2}^{1} \\
\dot{x}_{3}^{1}=-x_{3}^{1}-\frac{x_{2}^{1}}{1+\left(x_{2}^{1}\right)^{2}}-\frac{x_{2}^{2}}{1+\left(x_{2}^{2}\right)^{2}}+u+\varepsilon_{1}(t) \\
y_{1}=x_{1}^{1}
\end{array}\right. \\
& \left\{\begin{array}{l}
\dot{x}_{1}^{2}=x_{2}^{2}-x_{2}^{1} x_{3}^{1}-x_{1}^{2}+u x_{3}^{1} \\
\dot{x}_{2}^{2}=-x_{2}^{2}-\frac{x_{2}^{1}}{1+\left(x_{2}^{1}\right)^{2}}+\frac{x_{3}^{1}}{1+\left(x_{3}^{1}\right)^{2}}-u+\varepsilon_{2}(t) \\
y_{2}=x_{1}^{2}
\end{array}\right.
\end{aligned}
$$

where $u(t)=5 \sin (2 \pi t), \varepsilon_{1}(t)=0.1 \cos (t)$ and $\varepsilon_{2}(t)=0.5$ $\sin (t)$.
It is worth noticing that system (54)-(55) is in the form (1), with $q=2, p_{1}=p_{2}=1, \lambda_{1}=3$ and $\lambda_{2}=2$, and is not included in the classes of systems considered in [21,19]. Moreover, the state trajectory of the system is bounded and Assumption 1 holds (see Remark 3.1).

The corresponding observer (35) is given by

$$
\begin{aligned}
\left(\begin{array}{l}
\hat{x}_{1}^{1} \\
\hat{x}_{2}^{1} \\
\hat{x}_{3}^{1}
\end{array}\right)= & \left(\begin{array}{c}
\hat{x}_{2}^{1} \\
\hat{x}_{3}^{1} \\
0
\end{array}\right)+\left(\begin{array}{c}
-x_{1}^{1}+x_{1}^{2} u \\
-\hat{x}_{2}^{1} \\
-\hat{x}_{3}^{1}-\frac{\hat{x}_{2}^{1}}{1+\left(\hat{x}_{2}^{1}\right)^{2}}-\frac{\hat{x}_{2}^{2}}{1+\left(\hat{x}_{2}^{2}\right)^{2}}+u
\end{array}\right) \\
& -\theta^{\delta_{1}} \Delta_{1}^{-1}(\theta) S_{1}^{-1} C_{1}^{T}\left(\hat{x}_{1}^{1}-y_{1}\right) \\
\left(\begin{array}{c}
\hat{x}_{1}^{2} \\
\hat{x}_{2}^{2}
\end{array}\right)= & \left(\begin{array}{c}
\hat{x}_{2}^{2} \\
0
\end{array}\right)+\left(\begin{array}{c}
-\hat{x}_{2}^{1} \hat{x}_{3}^{1}-x_{1}^{2}+u \hat{x}_{3}^{1} \\
-\hat{x}_{2}^{2}-\frac{\hat{x}_{2}^{1}}{1+\left(\hat{x}_{2}^{1}\right)^{2}}+\frac{\hat{x}_{3}^{1}}{1+\left(\hat{x}_{3}^{1}\right)^{2}}-u
\end{array}\right) \\
& -\theta^{\delta_{2}} \Delta_{2}^{-1}(\theta) S_{2}^{-1} C_{2}^{T}\left(\hat{x}_{1}^{2}-y_{2}\right)
\end{aligned}
$$

where

$S_{1}^{-1} C_{1}^{T}=\left(\begin{array}{l}3 \\ 3 \\ 1\end{array}\right)$ and $S_{2}^{-1} C_{2}^{T}=\left(\begin{array}{l}2 \\ 1\end{array}\right)$ according to (23)

and

$\Delta_{1}^{-1}(\theta)=\operatorname{diag}\left(1, \theta^{\delta_{1}}, \theta^{2 \delta_{1}}\right)$ and

$\Delta_{2}^{-1}(\theta)=\operatorname{diag}\left(1, \theta^{\delta_{2}}\right)$ according to (12).

The values of $\delta_{1}$ and $\delta_{2}$ are computed according to (13) and are $\delta_{1}=\delta_{2}=1$.

The observer (56)-(57) has been simulated using the data, corresponding to the input $u$ and output measurements $y_{1}$ and $y_{2}$, issued from simulation of system (54)-(55). The simulation has been carried out with an observer gain $\theta=12$ using the following initial values $\hat{x}_{1}^{1}(0)=\hat{x}_{2}^{1}(0)=x_{i}^{k}(0)=0 k=1,2$ and $i=$ $1, \ldots, \lambda_{k} ; \hat{x}_{2}^{1}(0)=\hat{x}_{3}^{1}(0)=\hat{x}_{2}^{2}(0)=0.5$. Of practical interest, each measured variable has been corrupted by an additive Gaussian noise with zero mean value and a standard deviation equal to 0.1 . The underlying noise realizations are given in Fig. 1.

Fig. 2 shows the true time evolutions of the state variables $x_{2}^{1}, x_{3}^{1}$ and $x_{2}^{2}$ (issued from model simulation) with their respective estimates provided by the observer. Note the good agreement between the simulated and estimated values of the state variables in spite of the presence of the uncertainties $\varepsilon_{1}(t)$ and $\varepsilon_{2}(t)$ as well as noise measurements.

\section{Conclusion}

This paper presents a high gain observer design for a large class nonlinear MIMO systems including some uncertainties. The main contribution consists in relaxing the long standing triangular 

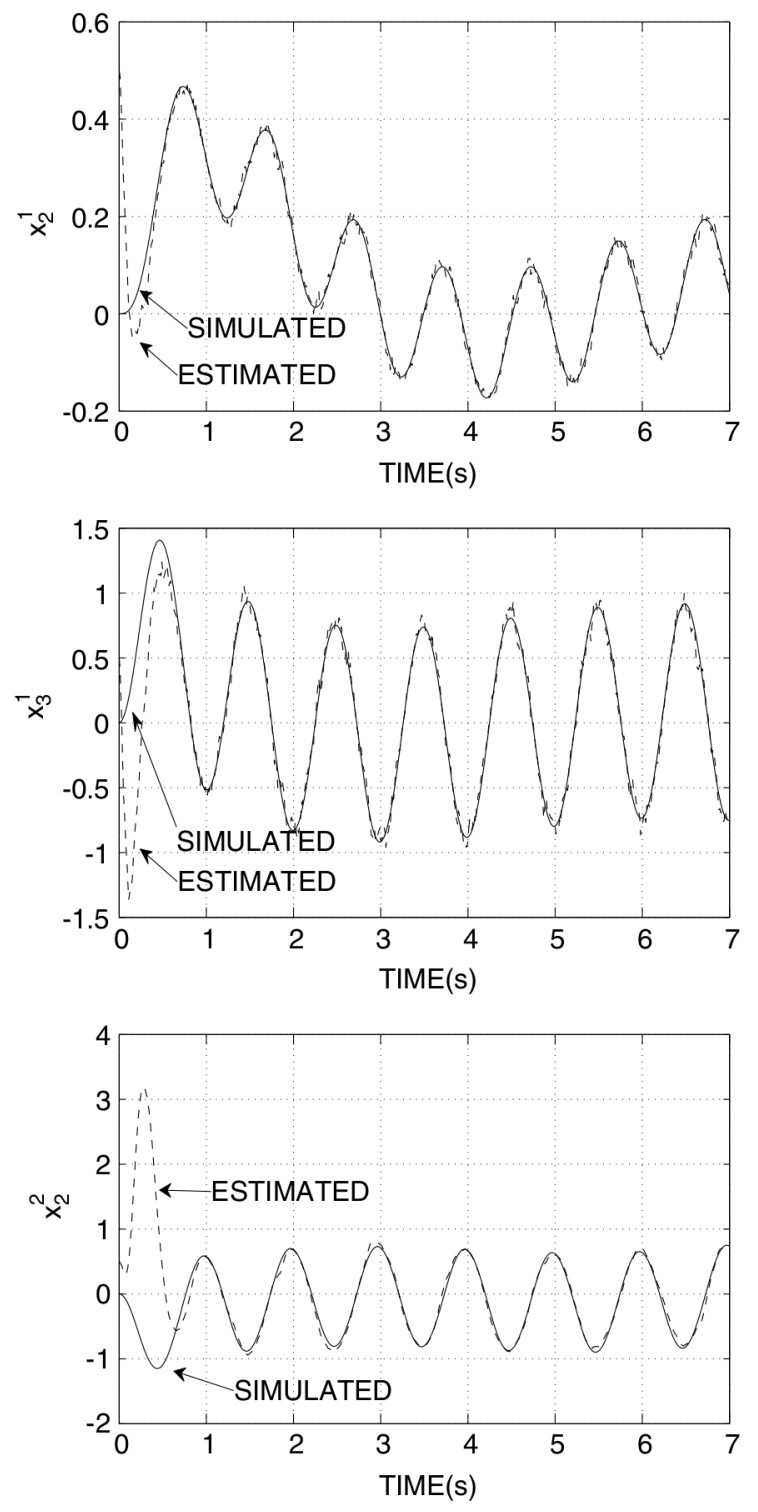

Fig. 2. Comparison of the simulated and estimated non-measured states.

structure assumption. Indeed, the class of systems is composed of cascade subsystems such that the time derivative of the last component of each subsystem may depend on the whole state of the system. Moreover, each subsystem is associated with a subset of the outputs. In the absence of uncertainties, one recovers a canonical form that characterizes a large class of nonlinear systems that are observable for any input, namely, those particular forms considered in [19,21].

From a fundamental point of view, it is shown that the observation error converges exponentially to zero in the absence of uncertainties and can be made arbitrarily small by properly specifying the observer gain design parameter when the uncertainties are considered. Simulation results have been reported to illustrate the proposed observer accuracy performances in the presence of uncertainties and noise measurements.

The account for uncertainties is particularly motivated by incoming studies on the observer design for systems with unknown inputs. Let us however emphasize that the problem of seeking state transformations, that bring systems that are observable for any input into the form (1), has not been investigated here and is still open.

\section{References}

[1] R.E. Kalman, R.S. Bucy, New results in linear filtering and prediction theory, J. Basic Eng. (1961) 95-108.

[2] A.J. Krener, A. Isidori, Linearization by output injection and nonlinear observers, Systems Control Lett. 3 (1983) 47-52.

[3] A.J. Krener, W. Respondek, Nonlinear observers with linearizable error dynamics, SIAM J. Control Optim. 23 (1985) 197-216.

[4] X.H. Xia, W.B. Gao, Nonlinear observer design by observer error linearization, SIAM J. Control Optim. 27 (1) (1989) 199-216.

[5] M. Hou, A.C. Pugh, Observer with linear error dynamics for nonlinear multioutput systems, Systems Control Lett. 37 (1999) 1-9.

[6] M. Guay, Observer linearization by output-dependent time-scale transformations, IEEE Trans. Automat. Control 47 (2002) 1730-1735.

[7] I. Souleiman, A. Glumineau, G. Schreirer, Direct transformation of nonlinear systems into state affine MISO form and nonlinear observers design, IEEE Trans. Automat. Control 48 (2003) 2191-2196.

[8] D. Boutat, A. Benali, H. Hammouri, K. Busawon, New algorithm for observer error linearization with a diffeomorphism on the outputs, Automatica 45 (2009) 2187-2193.

[9] R. Rajamani, Observers for Lipschitz nonlinear systems, IEEE Trans. Automat. Control 43 (3) (1998) 397-401.

[10] A. Zemouche, M. Boutayeb, A unified $H_{\infty}$ adaptive observer synthesis method for a class of systems with both Lipschitz and monotone nonlinearities, Systems Control Lett. 58 (2009) 282-288.

[11] M. Arcak, P. Kokotović, Nonlinear observers: a circle criterion design and robustness analysis, Automatica 37 (2001) 1923-1930.

[12] J.P. Gauthier, G. Bornard, Observability for any $u(t)$ of a class of nonlinear systems, IEEE Trans. Automat. Control 26 (1981) 922-926.

[13] J.P. Gauthier, H. Hammouri, S. Othman, A simple observer for nonlinear systems-application to bioreactors, IEEE Trans. Automat. Control 37 (1992) $875-880$.

[14] F. Deza, E. Busvelle, J.P. Gauthier, Exponentially converging observers for distillation columns and internal stability for the dynamic output feedback Chem. Eng. Sci. 47 (1992) 3935-3941.

[15] J.P. Gauthier, I.A.K. Kupka, Observability and observers for nonlinear systems, SIAM J. Control Optim. 32 (1994) 975-994.

[16] J. Rudolph, M. Zeitz, A block triangular nonlinear observer normal form, Systems Control Lett. 23 (1994) 1-8.

[17] K. Busawon, M. Farza, H. Hammouri, Observer design for a special class of nonlinear systems, Internat. J. Control 71 (1998) 405-418.

[18] M. Hou, K. Busawon, M. Saif, Observer design based on triangular form generated by injective map, IEEE Trans. Automat. Control 45 (7) (2000) 1350-1355.

[19] H. Shim, Y.I. Son, J.H. Seo, Semi-global observer for multi-output nonlinear systems, Systems Control Lett. 42 (2001) 233-244.

[20] M. Farza, M. M'Saad, L. Rossignol, Observer design for a class of MIMO nonlinear systems, Automatica 40 (2004) 135-143.

[21] H. Hammouri, M. Farza, Nonlinear observers for locally uniformly observable systems, ESAIM J. Control Optim. Calc. Var. 9 (2003) 353-370.

[22] N. Kazantzis, C. Kravaris, Nonlinear observer design using Lyapunov's auxiliary theorem, Systems Control Lett. 34 (1998) 241-247.

[23] M. Fliess, C. Join, H. Sira-Ramirez, Nonlinear estimation is easy, Int. J. Model. Ident. Control 4 (1) (2008) 12-27.

[24] L. Jaulin, Robust set-membership state estimation; application to underwater robotics, Automatica 45 (2009) 2002-2006.

[25] M. Farza, M. Triki, T. Maatoug, M. M'Saad, B. Dahhou, Unknown inputs observers for a class of nonlinear systems, in: Proc. of the 10th Int. Conf. of Sciences \& Techniques of Automatic, Hammamet, Tunisia, 2009.

[26] G. Bornard, H. Hammouri, A high gain observer for a class of uniformly observable systems, in: Proc. 30th IEEE Conference on Decision and Control, vol. 122, Brighton, England, 1991.

[27] G. Bornard, H. Hammouri, A graph approach to uniform observability of nonlinear multi output systems, in: Proc. of the 41st IEEE Conference on Decision and Control, Las Vegas, Nevada, USA, 2002.

[28] Y. Shena, X. Xia, Semi-global finite-time observers for nonlinear systems, Automatica 44 (2008) 3152-3156.

[29] H. Shim, A passivity-based nonlinear observer and a semi-global separation principle, Ph.D. Thesis, School of Electrical Engineering, Seoul National University, 2000. 